Memorias del VII Encuentro Nacional de Experiencias en la Enseñanza de la Biología y la Educación Ambiental y II Congreso Nacional de Investigación en la Enseñanza de la Biología

\title{
ENSEÑANZA DE COMPETENCIAS DE PENSAMIENTO CIENTÍFICO POR RESOLUCIÓN DE SITUACIONES PROBLEMATIZADORAS A FUTUROS DOCENTES DE CIENCIAS NATURALES
}

\section{TEACHING SCIENTIFIC THINKING SKILLS BY SOLVING SITUATIONS TO FUTURE TEACHERS PROBLEMATIZING OF NATURAL SCIENCES}

\author{
Mabel Tatiana Torrente Díaz ${ }^{1}$ \\ Wilson Armando Guevara ${ }^{2}$ \\ Sonia Echeverry Hernández ${ }^{3}$ \\ Elías Francisco Amórtegui Cedeño ${ }^{4}$
}

\section{Resumen}

Se presenta una propuesta de investigación acerca de la formación de profesores de Ciencias Naturales al interior del semillero VIRHOBAC adscrito al Grupo de investigación y Pedagogía en Biodiversidad y al semillero ENCINA adscrito al Grupo Conocimiento Profesional del Profesor, pertenecientes al programa de Licenciatura en Ciencias Naturales de la Universidad Surcolombiana (Neiva-Huila). La propuesta nace de la experiencia de práctica pedagógica en la que se pudo reconocer que dentro del proceso de enseñanza de las ciencias naturales y la educación ambiental deben considerar la formación de personas a través de los contenidos conceptuales, procedimentales y actitudinales para desenvolverse en su contexto cotidiano; sin embargo, es evidente la falta de una formación integral, puesto que la mayoría de los estudiantes consideran que la ciencia que aprenden en el colegio no es más que un requisito, debido a una posible falta de motivación por parte de los mismos y de sus maestros, además de la enseñanza rutinaria propia de un modelo conductista lo que no les permite desarrollar un pensamiento científico. Por eso, es preciso que los actuales y futuros docentes construyan y apliquen competencias de pensamiento científico, teniendo en cuenta que éstas les permiten acercar a los estudiantes a una visión del mundo científico más real,

\footnotetext{
${ }^{1}$ Estudiante IX semestre de Licenciatura en Educación Básica con énfasis en Ciencias Naturales y Educación Ambiental. Semillero de investigación Virhobac y Encina. Grupos de Investigación y Pedagogía en Biodiversidad y Conocimiento Profesional del Profesor de Ciencias. tatitodi@hotmail.com

${ }^{2}$ Estudiante $X$ semestre de Licenciatura en Educación Básica con énfasis en Ciencias Naturales y Educación Ambiental. Semillero de investigación Virhobac y Encina. Grupos de Investigación y Pedagogía en Biodiversidad y Conocimiento Profesional del Profesor de Ciencias. wilsyn826@hotmail.com

${ }^{3}$ Docente planta tiempo completo programa de Licenciatura en Educación Básica con énfasis en Ciencias Naturales y Educación Ambiental. Coordinadora de Semillero de investigación Virhobac. sonia.echeverry@usco.edu.co

${ }^{4}$ Docente planta tiempo completo programa de Licenciatura en Educación Básica con énfasis en Ciencias Naturales y Educación Ambiental. Coordinador de práctica pedagógica. Coordinador Semillero Encina y Grupo de Investigación Conocimiento Profesional del Profesor de Ciencias. elias.amortegui@usco.edu.co
} 
Bio-grafia Escritos sobre la Biologia y su Enseñanza.

Edición Extra-Ordinaria. ISSN 2027-1034 P.p 885 - 894

Memorias del VII Encuentro Nacional de Experiencias en la Enseñanza de la Biología y la Educación Ambiental y II Congreso Nacional de Investigación en la Enseñanza de la Biología

más accesible. Por esto, es conveniente enseñar a los futuros docentes a desarrollar dichas competencias, implementando una estrategia de resolución de situaciones problematizadoras y la elaboración de materiales de enseñanza científica con fundamento teórico y contextualizado.

\section{Abstract}

We present a research proposal on the training of teachers of Science into the nursery attached to VIRHOBAC and Pedagogy Research Group on Biodiversity and the nursery attached to OAK Teacher Professional Knowledge Group, belonging to the degree program in Natural Sciences Surcolombiana University (Neiva-Huila). The proposal stems from the experience of teaching practice in which he recognized that within the process of natural science education and environmental education should consider training people through the conceptual, procedural and attitudinal to function in its context every day, but there is a lack of comprehensive training, since most students view science learning in school is just a requirement, due to a possible lack of motivation from them and of their teachers, in addition to routine education own behavioral model which does not allow them to develop scientific thinking. Therefore, it is necessary for current and future teachers build and apply scientific thinking skills, considering that these allow them to bring students to a scientific worldview more real, more accessible. Therefore, it is desirable to teach future teachers develop these skills, implementing a resolution strategy problematizing situations and developing teaching materials scientific rationale and contextualized.

Palabras Clave: Situaciones problematizadoras, competencias de pensamiento científico, Ciencias naturales, microbiología, contextualización.

Keywords: Situations problematizing, scientific thinking skills, natural sciences, microbiology, contextualization

\section{Introducción}

La Universidad Surcolombiana con sede en Neiva- Huila, es un lugar de formación integral de ciudadanos a través de la asimilación, producción, aplicación y difusión de conocimientos científico, humanístico, tecnológico, artístico y cultural, con espíritu crítico, con el fin de que se aborden eficazmente la solución de los problemas del desarrollo humano integral de la región Surcolombiana con proyección nacional e internacional.

La institución cuenta con un programa adscrito a la Facultad de Educación con el nombre de Licenciatura en Ciencias Naturales y Educación Ambiental, con un plan de estudios que considera nueve semestres de formación profesional y ciento 
Memorias del VII Encuentro Nacional de Experiencias en la Enseñanza de la Biología y la Educación Ambiental y II Congreso Nacional de Investigación en la Enseñanza de la Biología

cincuenta y nueve créditos, de los cuales sólo cuarenta y siete son destinados a la formación pedagógica (Didáctica, Práctica pedagógica, componentes de Facultad), ciento doce de las áreas del componente básico específico (Física, Química y Biología) y del componente complementario flexible (Electivas institucionales o del programa).

La práctica pedagógica es el espacio que brinda el programa de Licenciatura en Ciencias Naturales y Educación Ambiental conjuntamente con las instituciones educativas, preferiblemente de carácter público del departamento del Huila, para la formación inicial de docentes; como lo dice el reglamento de prácticas profesionales 2011, "es un proceso de construcción curricular -acción- reflexión e investigación- que rompe con la concepción tradicional de la práctica, como el simple entrenamiento bajo un estado de subordinación del practicante", Programa de Licenciatura en Ciencias Naturales y Educación ambiental, (2011). Este proceso se desarrolla en los dos últimos semestres del programa (octavo y noveno).

Durante el desarrollo de la práctica pedagógica I realizada durante el primer semestre del año 2013, se evidenció que uno de los problemas propios de la formación docente es la falta de herramientas, métodos, estrategias generales y específicas para la enseñanza de los contenidos, debido a que el programa ha centrado la formación de sus futuros docentes en los componentes disciplinares, olvidando dos de los objetivos establecidos para su formación pedagógica: "Formar un educador en Ciencias Naturales y Educación Ambiental competente para ejercer la enseñanza de las Ciencias Naturales de forma crítica y reflexiva con el propósito de mejorar permanentemente la enseñanza que imparte, además de poder desarrollar proyectos mediante el trabajo en equipo o trabajo integrado" PEP, (2008) y "Formar educadores con una visión integral de las Ciencias Naturales y con elementos didácticos para contextualizar, mediante la organización de los contenidos, el conocimiento científico con la problemática de la vida cotidiana y permitir que los estudiantes construyan las teorías, conceptos y principios generales de las disciplinas científicas que conforman las Ciencias Naturales" PEP, (2008). Así como lo dice Angulo, (2002) "La principal influencia en el desarrollo profesional de los profesores de ciencias naturales es la forma en que han sido formados en las instituciones de educación superior".

Dentro del programa existe un Grupo de Investigación y Pedagogía en Biodiversidad (GIPB) que está conformado por un grupo de docentes y estudiantes que realizan investigaciones en las diferentes áreas del conocimiento científico, con el fin de contribuir al perfeccionamiento de las actividades pedagógicas investigativas de la Universidad Surcolombiana.

El grupo actualmente cuenta con cuatro semilleros de investigación, todos enfocados a la investigación en Biodiversidad y Pedagogía a nivel regional y 
Bio-grafia Escritos sobre la Biologia y su Enseñanza.

Edición Extra-Ordinaria. ISSN 2027-1034 P.p 885 - 894

Memorias del VII Encuentro Nacional de Experiencias en la Enseñanza de la Biología y la Educación Ambiental y 11 Congreso Nacional de Investigación en la Enseñanza de la Biología

nacional; uno de los semilleros de investigación, es VIRHOBAC, grupo que tiene como objetivo formar investigadores en el campo disciplinar y pedagógico de la Microbiología; el semillero cuenta con diferentes líneas de estudio: investigación de la microbiología de aguas, de suelos, del aire, de alimentos, clínica y de enseñanza de la misma. En particular esta última línea, tiene como objetivo estudiar las formas de enseñanza, aprendizaje y comunicación de saberes científicos propios de esta ciencia.

Para el caso de esta propuesta, la muestra será el curso de Microbiología que hace parte del componente especifico de la carrera, se orienta en el segundo semestre y es requisito para ingresar al curso de Botánica y así continuar la línea de la Biología; la Microbiología cuenta con un microdiseño que contempla la formación en contenidos conceptuales y procedimentales.

Desde este punto de vista, se reconoce que es totalmente necesario que un profesor tenga un buen manejo de los contenidos conceptuales propios de su profesión, debido a que, sin importar la metodología o el modelo pedagógico que utilice, el docente es quien elige la temática que necesita enseñar al estudiante de acuerdo con las capacidades, actitudes o habilidades que quiera desarrollar en él; por esta razón, es importante que el profesor conozca los contenidos conceptuales de las disciplinas. Así como lo afirman algunos investigadores Izquierdo, (2000), Adúriz-Bravo e Izquierdo, (2002), Angulo, (2002) que las insuficiencias en la preparación, actualización sistemática y contextualizada del profesor de ciencia en cuanto a los contenidos científicos de la materia a enseñar es una primera dificultad que puede limitar gravemente el potencial innovador de cualquier profesor.

Ésta disciplina presenta dificultades de aprendizaje y desarrollo de competencias en los estudiantes, debido a varias razones entre las cuales se pueden enunciar: el uso de un modelo pedagógico no adecuado por parte del profesor, como es el caso del modelo "tradicionalista" en donde el estudiante es un actor secundario en el proceso de Enseñanza-Aprendizaje, limitándose a memorizar los conceptos sin contextualización ni uso de los mismos y sin motivación alguna, originando en el estudiante conocimientos acerca de los temas de la disciplina, pero que no puede utilizar (Contextualizar) en su vida cotidiana y desaparecen con el paso del tiempo.

Al realizar una revisión de los antecedentes relacionados con temáticas de desarrollo de competencias de pensamiento científico y enseñanza por resolución de problemas, se encontró que a nivel institucional se han publicado dos artículos realizados: uno por Rivera, (2009) y Narváez, (2009) estos artículos muestran cómo la aplicación de la metodología basada en resolución de problemas es una alternativa de enseñanza-aprendizaje constructivista favorable, ya que contextualizaron el entorno en el que se encontraban los estudiantes y realizaron 
Bio-grafia Escritos sobre la Biologia y su Enseñanza.

Edición Extra-Ordinaria. ISSN 2027-1034 P.p 885 - 894

Memorias del VII Encuentro Nacional de Experiencias en la Enseñanza de la Biología y la Educación Ambiental y II Congreso Nacional de Investigación en la Enseñanza de la Biología

situaciones problematizadoras para que los estudiantes desarrollaran una serie de conceptos acerca de la geología y la química respectivamente.

A nivel nacional (Colombia), se encuentran nueve trabajos de investigación, la mayoría realizados en universidades públicas enfocadas en la pedagogía y la didáctica científica; así que revisamos cada uno de ellos, con el fin de elegir los más cercanos a nuestro trabajo de investigación entre estos fueron: García y Ladino, (2008), Basto y Garcia, (2007), Franco, (2011), Torres y Pantoja, (2012), Murillo et al. (2012), Vargas, (2010), Torres, (2012), Fonseca, (2010), estos trabajos, investigaciones, ponencias y propuestas de investigación consultadas describen la necesidad de implementar en la enseñanza de las Ciencias Naturales una nueva estrategia de aprendizaje en la que los conceptos o los contenidos conceptuales, estén ligados a la realidad; es decir, a un contexto especifico de su vida, además muestran como la implementación de una estrategia didáctica enfocada en la resolución de problemas puede llegar a potencializar las habilidades cognitivas, procedimentales y actitudinales, en algunos de estos implementaron herramientas como: cartillas para el desarrollo de competencias de pensamiento científico, otros trabajaron en torno a las Tic, uso de museos, elaboración de prácticas de laboratorio artesanales, entre otras; pero todas buscando el mismo propósito que era precisamente la evolución conceptual. Además muestran la importancia de que los docentes conozcan acerca de las competencias de pensamiento científico, inmersas en una ciencia netamente humanizadora, con el fin de que las puedan reconocer y desarrollar en cada uno de sus estudiantes, para formar no un científico; sino un ciudadano capaz de solucionar cualquier situación problema a la que se enfrente en su vida cotidiana.

A nivel internacional, se gestionaron y se gestionan actualmente muchos trabajos de investigación especialmente en países como España, Costa Rica, Argentina y Chile, siendo éstos los países en donde se encuentran la mayor parte de autores que son referenciados en la presente propuesta. Algunos de ellos son: Zúñiga et al. (2011), Quintanilla et al. (2010), Labarrere et al. (2010), estos trabajos muestran cómo la metodología basada en resolución de problemas científicos (RPC) pueden generar competencias de pensamiento científico (CPC) en estudiantes con diversidad cultural.

Dentro de los antecedentes consultados, no se encuentra antecedente alguno donde se enseñe a generar competencias de pensamiento científico a profesores y/o a futuros profesores, a través de situaciones problematizadoras.

La enseñanza-Aprendizaje es un proceso complejo que requiere de todas las capacidades del individuo, tanto del que aprende como del que enseña, siempre que se quiera tener un desarrollo total, completo y real del mismo. Así, el desarrollo de competencias de pensamiento científico en cualquier individuo, evidencia un uso real de las capacidades de los involucrados en éste proceso. 
Bio-grafia Escritos sobre la Biologia y su Enseñanza.

Edición Extra-Ordinaria. ISSN 2027-1034 P.p 885 - 894

Memorias del VII Encuentro Nacional de Experiencias en la Enseñanza de la Biología y la Educación Ambiental y II Congreso Nacional de Investigación en la Enseñanza de la Biología

Pero, ¿Qué son las competencias de pensamiento científico?; las competencias de pensamiento científico como las plantea Quintanilla et al. (2010), son una "combinación dinámica de atributos", tales como habilidades, actitudes, destrezas, valores y responsabilidades en relación con conocimientos que desarrolla un individuo para un aprendizaje real, entendiéndose éste como una comprensión de la ciencia "de manera no reproductiva" (Quintanilla et al. 2010), el desarrollo de dichas competencias permiten que el individuo se pueda "integrar a la sociedad" de forma competente y con responsabilidad social, en donde está en la capacidad de ofrecer explicaciones -de forma no imitativa- dinámicas y entendibles sobre conocimiento científico.

\section{Metodología Propuesta}

La investigación que se realizará es de tipo cualitativo descriptivo y se desarrollará teniendo en cuenta las capacidades que tiene el ser humano para dar solución a los problemas de la vida, buscando el desarrollo del mencionado pensamiento científico a través de situaciones problematizadoras del común, enfocadas a las temáticas abordadas en el curso de Microbiología.

La investigación se llevará a cabo durante seis meses, desarrollando las siguientes etapas: etapa I, comprendida por las fases a) Búsqueda bibliográfica relacionada con temas como las competencias de pensamiento científico y resolución de problemas, fase b) Diseño, aplicación y validación de un cuestionario como diagnóstico sobre conocimientos de la asignatura de Microbiología y enseñanza de la misma aplicada a los estudiantes que cursan dicha asignatura en el segundo semestre de la Licenciatura en Ciencias Naturales y Educación ambiental de la Universidad Surcolombiana, considerados como el grupo de estudio.

En la etapa II, las fases a desarrollar son: a) Enseñanza mediante un seminario que comprende los siguientes contenidos: concepción de ciencia, Microbiología, competencias de pensamiento científico, situaciones problematizadoras y diseño de situaciones problematizadoras para desarrollar competencias de pensamiento científico en Microbiología dirigidos al grupo de estudio, fase b) Aplicación de la estrategia de enseñanza por parte de los futuros docentes en Instituciones Educativas de la ciudad de Neiva y fase c) Aplicación de un cuestionario validado por expertos, para evaluar la estrategia de enseñanza de la microbiología, a partir de la resolución de situaciones problematizadoras, donde Las competencias de pensamiento científico generadas, se evaluarán por el método de triangulación de datos.

En la etapa III, las fases a desarrollar son: a) Sistematización y categorización de la información recopilada, b) Análisis de los resultados y c) Divulgación de los resultados a partir de la elaboración de un artículo científico y de una unidad 
Bio-grafia Escritos sobre la Biologia y su Enseñanza.

Edición Extra-Ordinaria. ISSN 2027-1034 P.p 885 - 894

Memorias del VII Encuentro Nacional de Experiencias en la Enseñanza de la Biología y la Educación Ambiental y 11 Congreso Nacional de Investigación en la Enseñanza de la Biología

didáctica recopilando todo el diseño de las situaciones problematizadoras que realizaron los estudiantes del curso de Microbiología.

\section{Resultados y discusión}

Se ha evidenciado la necesidad de articular el conocimiento científico con el contexto o el ambiente en el cual se desenvuelven los estudiantes, según su entorno social y así formar ciudadanos con pensamiento científico, aquel que se identifica como la evolución conceptual; así que es necesario enseñar a los estudiantes no a resolver problemas científicos, sino a enseñarles cómo enfrentarse a la resolución de problemas científicos (Quintanilla 2010).

Para ello es importante reconocer ¿Qué modelo de ciencia se enseña y qué modelo de ciencia aprenden los estudiantes?. La ciencia de hoy no es la misma de hace un siglo donde se enseñaban únicamente las teorías y conceptos de la misma; hoy en día se debe buscar la re significación de ciencia, de manera que esta tenga un enfoque netamente humanizador, que se pueda aplicar a la vida con el fin de formar ciudadanos competentes, capaces de resolver cualquier situación utilizando unas habilidades, destrezas, metodologías, entre otras. Así como lo afirma el profesor Quintanilla, M (2010) "La ciencia como una actividad humana, con finalidades humanas, un concepto que esté inserto en el mundo, con un componente ético, que cuente con una manera de interpretar el mundo con teorías sólidas"

El programa de Licenciatura en Ciencias Naturales y educación ambiental es un programa vinculado a la Facultad de Educación, es donde se forman los futuros docentes y justamente es el espacio indicado para enseñarles a estos futuros profesionales a desarrollar las competencias de pensamiento científico. Teniendo en cuenta estos argumentos, surge una pregunta ¿Cuáles competencias de pensamiento científico identifican y proponen los futuros docentes de Licenciatura en Ciencias Naturales y Educación Ambiental, específicamente del curso de Microbiología utilizando la estrategia resolución de situaciones problematizadoras? Para dar solución a esta pregunta, se propone como objetivo general: Enseñar por medio de un seminario a identificar y generar competencias de pensamiento científico utilizando la estrategia didáctica resolución de situaciones problematizadoras a los futuros docentes de Ciencias Naturales y Educación Ambiental del curso de Microbiología de la Universidad Surcolombiana.

Dentro de los objetivos específicos están: a) Diseñar, validar y aplicar un instrumento para identificar las concepciones acerca de enseñanza de la microbiología, las competencias de pensamiento científico y enseñanza por resolución de problemas, que tienen los estudiantes del curso de microbiología de la Universidad Surcolombiana, b) Diseñar y aplicar un seminario para la enseñar a los futuros docentes de Ciencias Naturales a identificar y generar competencias de 
Bio-grafia Escritos sobre la Biologia y su Enseñanza.

Edición Extra-Ordinaria. ISSN 2027-1034 P.p 885 - 894

Memorias del VII Encuentro Nacional de Experiencias en la Enseñanza de la Biología y la Educación Ambiental y II Congreso Nacional de Investigación en la Enseñanza de la Biología

pensamiento científico utilizando la estrategia didáctica: Resolución de situaciones problematizadoras, c) Contribuir a la formación integral de los futuros maestros de Ciencias Naturales, con prácticas docentes desde el inicio de sus carreras profesionales, d) Contribuir al desarrollo de competencias científicas en escolares de educación básica, con la metodología de situaciones problema, la utilización de la Microbiología como disciplina y su entorno cotidiano como contexto, e) Sistematizar, analizar y divulgar la información obtenida, mediante la generación de un artículo científico, para presentar a la comunidad una propuesta válida y confiable de enseñanza.

\section{Conclusiones}

Después de la implementación de la estrategia y del proyecto en general, se espera que los estudiantes del curso de Microbiología del programa de Licenciatura en Ciencias Naturales y Educación ambiental adquieran en su formación académica una estrategia alternativa de enseñanza- aprendizaje para desarrollar en su vida profesional. Además, promover en los estudiantes de Educación Básica y media de las instituciones educativas intervenidas habilidades, destrezas, valores, responsabilidades, que les permita de manera completa, idónea y sin imitación, explicar "conocimiento científico" y comprender la ciencia, específicamente conocimientos propios de la Microbiología, que se resume en desarrollo de competencias de pensamiento científico (Quintanilla, 2010); lo anterior se conseguirá gracias a la aplicación de la estrategia didáctica: resolución de situaciones problematizadoras.

Se espera también adjuntar todas las situaciones problema contextualizadas en la disciplina de la Microbiología en una unidad didáctica, dirigida a toda la comunidad académica.

\section{Bibliografía}

-Angulo, F. (2002). Formulación de un modelo de autorregulación de los aprendizajes desde la formación profesional del biólogo y del profesor de biología. Tesis Doctoral. Facultad de Educación. Universidad Autónoma de Barcelona, España.

-Basto, D. M. \& Garcia, S. V. (2010). Desarrollo de competencias científicas y ciudadanas por medio de una estrategia basada en la resolución de problemas. Tesis para obtener el título de Licenciado en Educación Básica con énfasis en Ciencias Naturales y Educación Ambiental. Escuela de Educación. Facultad de Ciencias Humanas. Universidad Industrial de Santander, Bucaramanga, Colombia.

-Franco, R. A. (2011). Competencias científicas y resolución de problemas en el instituto pedagógico nacional. Año Internacional de la Química 2011. 26-33. 
Bio-grafia Escritos sobre la Biologia y su Enseñanza.

Edición Extra-Ordinaria. ISSN 2027-1034 P.p 885 - 894

Memorias del VII Encuentro Nacional de Experiencias en la Enseñanza de la Biología y la Educación Ambiental y II Congreso Nacional de Investigación en la Enseñanza de la Biología

-García, G. A. \& Ospina, Y. L. (2008). Desarrollo de competencias científicas a través de una estrategia de enseñanza y aprendizaje por investigación. Studiositas, 3, 7-16.

-Murillo, J., Mejía, L. M. \& Gómez, N. S. (2012). El desarrollo de competencias científicas: una propuesta que integra el museo de la universidad de Antioquia como recurso didáctico, en la metodología del aprendizaje basado en problemas. Revista EDUCYT 2012. 36-57.

-Narváez, L. J. (2009). Aprendizaje significativo de algunos conceptos químicos, a través de resolución de problemas. Entornos, 21, 43-56.

-Página Web Universidad Surcolombiana. Misión-Visión. Documento consultado el día 10 de agosto del 2013 en: http://usco.edu.co/pagina/mision-y-vision.

-Página Web Universidad Surcolombiana. Programa de Licenciatura en Educación Básica con énfasis en Ciencias Naturales y Educación Ambiental. Objetivos. Documento consultado el día 10 de agosto del 2013 en: http://usco.edu.co/pagina/ciencias-naturales.

-Quintanilla, M., Joglar, C., Jara, R., Camacho, J., Ravanal, E., Labarrere, A., Cuellar, L., Izquierdo, M., \& Chamizo, J. (2010). Resolución de problemas científicos escolares y promoción de competencias de pensamiento científico. ¿Qué piensan los docentes de química en ejercicio? Revista Enseñanza de las Ciencias. 28(2). 185-198

-Quintanilla, M., Labarrere, A., Díaz, L., Santos, M., Ravanal, E., Cuellar, L., Camacho, J., Soto, F., Joglar, C., Jara, R. \& Ramirez, P. (2010). Identificación, caracterización y promoción de competencias de pensamiento científico mediante la resolución de problemas en estudiantado de secundaria. XIII Encuentros de Didáctica de las Ciencias Experimentales. Proyecto FONDECYT 1070795. Facultad de Educación. Pontificia Universidad Católica de Chile. G.R.E.C.I.A.

-Rivera, O. (2009). Aprendizaje significativo de algunos conceptos geológicos a través de resolución de problemas en estudiantes de quinto de primaria de la institución educativa departamental sede el lago. Neiva: Universidad Surcolombiana, Facultad de Educación.

-Torres, A. (2010). Una propuesta alternativa para el desarrollo de las competencias científicas en el aula de clase del área de ciencias naturales y educación ambiental. Ponencia. $2^{\circ}$ congreso Internacional de investigación y educación en ciencias y tecnología. EDUCYT. 
Edición Extra-Ordinaria. ISSN 2027-1034 P.p 885 - 894

Memorias del VII Encuentro Nacional de Experiencias en la Enseñanza de la Biología y la Educación Ambiental y 11 Congreso Nacional de Investigación en la Enseñanza de la Biología

-Torres, Á. (2012). El desarrollo de competencias científicas mediante el uso de estrategias didácticas basadas en la indagación. Revista EDUCYT. 98-118.

-Vargas, E. (2010). Desarrollo de competencias científicas en estudiantes de grado $9^{\circ}$ a partir del diseño e implementación de prácticas de laboratorio artesanales en microbiología. Tunja: Grupo de Estudios Pedagógicos en Microbiología Ambiental "MICRAM", Licenciatura en Ciencias Naturales y Educación Ambiental, Universidad Pedagógica Y Tecnológica De Colombia (UPTC).

-Zúñiga, A. \& Naranjo, J. A. (2011). Nivel de desarrollo de las competencias científicas en estudiantes de secundaria de (Mendoza) Argentina y (San José) Costa Rica. Revista Iberoamericana de Educación. 56/2. 1-12 